(C) [2008] IEEE. Reprinted, with permission from [Rachod Patachaianand and Kumbesan Sandrasegaran, Performance Study of Video Streaming in Commercial UMTS

Network, Proceedings of 2008 IEEE $8^{\text {th }}$ International Conference on Computer and Information Technology, CIT 2008]. This material is posted here with permission of the IEEE. Such permission of the IEEE does not in any way imply IEEE endorsement of any of the University of Technology, Sydney's products or services. Internal or personal use of this material is permitted. However, permission to reprint/republish this material for advertising or promotional purposes or for creating new collective works for resale or redistribution must be obtained from the IEEE by writing to pubs-permissions@ieee.org. By choosing to view this document, you agree to all provisions of the copyright laws protecting it 


\title{
Performance Study of Video Streaming in Commercial UMTS Network
}

\author{
Rachod Patachaianand and Kumbesan Sandrasegaran \\ Centre for Real-time Information Networks (CRIN) \\ University of Technology, Sydney (UTS) \\ Email: rapatach@eng.uts.edu.au
}

\begin{abstract}
This paper studies the performance of a real-time application in a live Universal Mobile Telecommunication System (UMTS) network. In the study, special user equipment (UE) capable of collecting performance parameters such as throughput and bit error rate (BER) was used. Two scenarios are considered in this paper: typical scenario when soft handover (SHO) is enabled and an extreme scenario when a situation of cell edge is created by forcing the terminal to strictly connect to one cell. The experiment results show various aspects of practical implementations and protocols which are currently used in live UMTS network. In the typical scenario, when a code with a large spreading factor $(S F)$ is in use, the required signal-tonoise ratio target (SIRT) is low. In the cell edge case, a code with high SF of 32 is used in a poor radio condition. There results are consistent with the relevant theory.
\end{abstract}

\section{Introduction}

Universal Mobile Telecommunication System (UMTS) is designed to deliver new applications such as video streaming and video conferencing. UMTS is based on Wideband Code Division Multiple Access (WCDMA) technology. This technology provides many powerful features to deliver high-bandwidth real-time applications over the radio channel. Such features include soft handover (SHO), data rate adaptation, and signal-to-noise ratio target (SIRT) adjustment, etc.

In WCDMA, the data rate over the air interface may be adapted by changing the spreading code in use. This function is typically performed by Radio Network Controller (RNC). The RNC has to measure the signal quality and load that each base station (or Node B) is serving, before assigning a code with the most appropriate spreading factor (SF) to each connection. This code may be changed if the radio condition changes, for example, when high block error rate (BLER) in a connection is detected.

There are many publications that have studied the performance of operating UMTS system. In [1], the number of cells in active set and basic power control functions are monitored. Authors in [2] measure SHO gain in the uplink direction. Performance of Voice over Internet Protocol (VoIP) in real UMTS network is evaluated in [3]. The antenna gains of using different antenna configurations are discussed in [4]. It can be seen that each paper focused on different aspects, and none of them focused on the performance of real time video services, which is the main focus on this paper.

This paper aims to study the performance of real-time mobile TV service in a commercial UMTS network. The paper is organised as follows: Section 2 explains the test environment in the experiment. Section 3 provides the experimental results. Section 4 concludes this paper.

\section{Streaming Services in UMTS}

UMTS services are classified into four classes: Conversational, Streaming, Interactive, and Background. Each class has different Quality of Service (QoS) requirements. For example, Conversational-Class services require guaranteed bit rate and minimum delays. Streaming Class services need guaranteed bit rate, but delay requirement is more relaxed.

Streaming services are new features that UMTS can provide. Use of this service is quickly becoming more and more popular as it offers the mobile phones users the ability to watch live TV programs on their mobile phones anywhere anytime [5]. In the service provider perspective, the existence of the Multimedia Broadcast/Multicast Service (MBMS) makes broadcast services such as TV streaming more attractive as it allows the network to support theoretically unlimited number of users with constant network load [5]. Therefore, a study of behavior of such service in real UMTS network is invaluable for research in this field.

\section{Test Environment}

The experiments were carried out in a commercial $3 \mathrm{G}$ network in Australia. This network offers both WCDMA and HSDPA services. Only WCDMA performance will be focused in this paper.

A special terminal built on Anite's technology with particular software (NEMO) was used to run applications and collect data. A number of applications may be run on the terminal and the software will run as background to log performance information such as throughput and BLER. The terminal is capable of measuring and collecting data only. The analysis of collected data has to be performed using particular software. In this paper, the measurement data were extracted using NEMO software, and all statistical analysis and presentations of the data were conducted using MATLAB.

The measurements were performed on a main street at night to ensure that any disturbance from other users was minimised. The user was pedestrian and using a real-time video-streaming service. There are two scenarios that are considered in this paper. The first scenario is a typical UMTS network environment i.e. soft handover is allowed. Another scenario is an extreme case when the terminal is 
at the edge of the cell and moving out of the coverage. This situation is emulated by forcing the terminal to strictly connect to one cell only i.e. any kind of handover is disabled.

\section{Experiment Results}

In this section, the performance of WCDMA with realtime video application is presented and analysed in two scenarios: with SHO (typical) and Cell edge.

\subsection{Mobile Live TV: Typical Scenario}
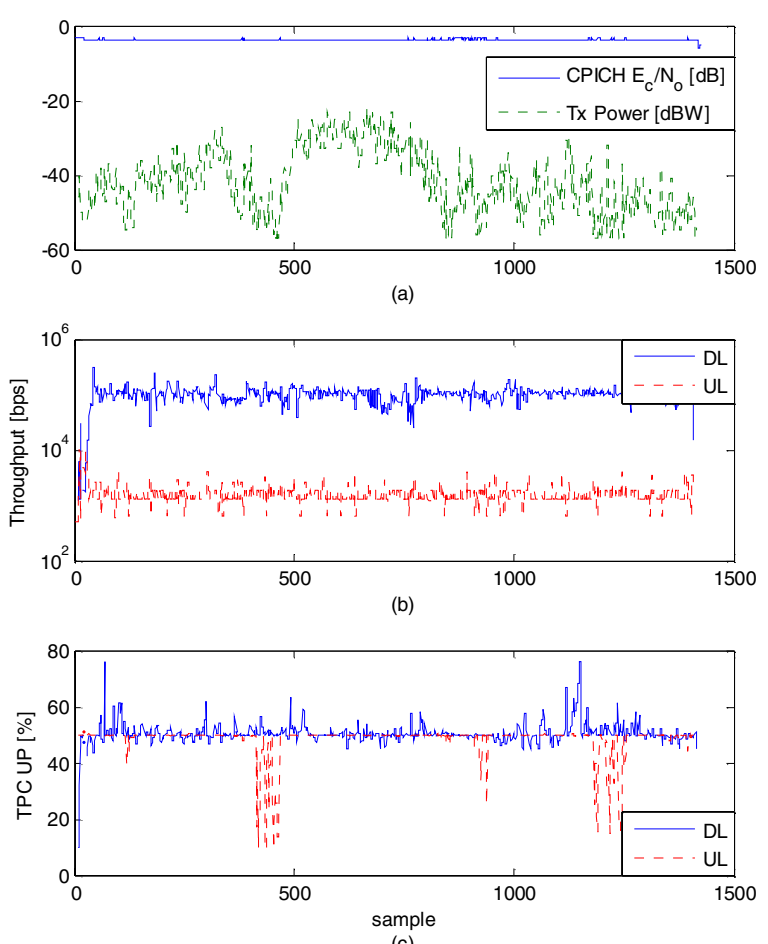

Figure 1 Performance of WCDMA real-time service

Figure 1 shows typical WCDMA performance indicators. Figure 1 (a) represents common pilot channel $(\mathrm{CPICH}) \mathrm{Ec} / \mathrm{No}$ in $\mathrm{dB}$ (solid line), and transmission power at $\mathrm{UE}$ in $\mathrm{dBW}$ (dotted line). Figure 1 (b) represents the throughput in downlink (solid line), and uplink (dotted line). Figure 1 (c) represents the percentage of Transmit Power Control (TPC) power up command in downlink (solid line), and uplink (dotted line). The $\mathrm{x}$ axis is measurement samples (around $300 \mathrm{~ms}$ per sample).

Figure 1 (a) illustrates that, with SHO, UE always connects to a Node B that provides the highest Ec/No. This will result in the minimum required transmission power to maintain the connection as shown in the figure that the required transmission power is just around -40 $\mathrm{dBW}$, which is well under the maximum of $-9 \mathrm{dBW}$ in a standard network dimensioning [6].

Figure 1 (b) shows that, in sprite of some variations, the throughput required to maintain the real-time videostreaming service could be provided. The statistical result given in Figure 2 indicates that $80 \%$ of the downlink throughput is greater than $80 \mathrm{kbps}$.

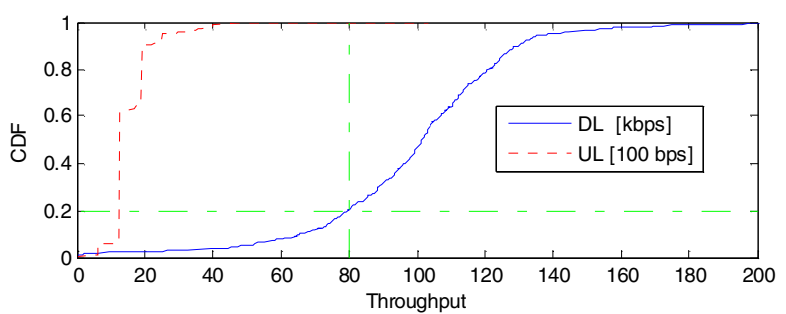

Figure 2 CDF of downlink and uplink throughput

In addition to basic parameters given in Figure 1 and Figure 2, more specific parameters such as SIR target, BLER, and adaptive SF are given in Figure 3.
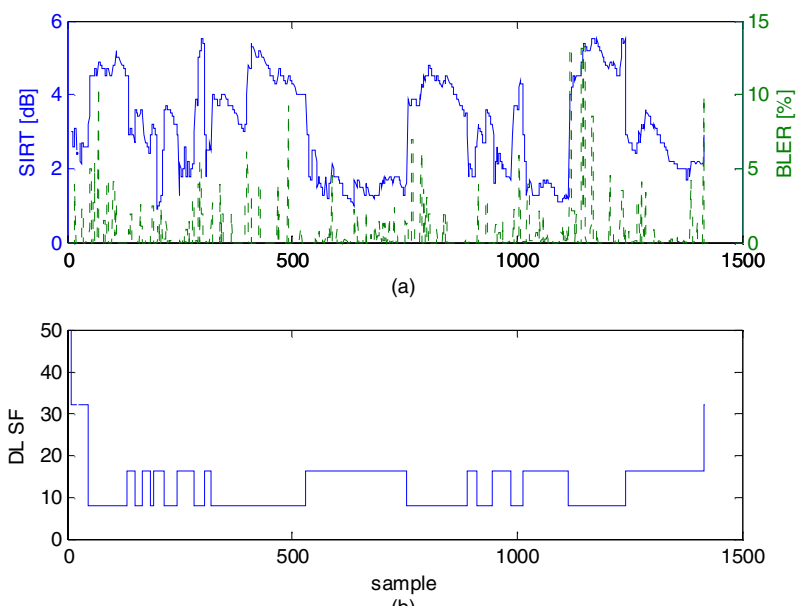

Figure 3 WCDMA adaptive parameters

Figure 3 (a) shows SIR $_{\text {target }}$ (solid line) and BLER (dotted line). Figure 3 (b) shows the spreading factor in use versus measurement samples (time).

According to the principle of outer loop power control, the SIRT should be increased if BLER is detected. However, in the real implementation, considering only BLER is not enough. That is, it is necessary for outer loop power control to take into account the SF in use in a particular period. It is shown in Figure 3 (a) and (b) that, with a high SF (such as between samples 520 and 700), a low SIRT is required. This is due to the fact that, with a high SF factor, the processing gain at the receiver is also high, implying more interference robustness. However, it is important to note that, although a code with high SF factor is more robust and requires less SIRT, it is not desirable. This is because lower throughput could be achieved with such code, and hence poorer quality of services. It was found from Figure 3 (b) and the fieldexperiment that the proportion of $\mathrm{SF}=8$ and $\mathrm{SF}=16$ was approximately the same, and good real-time video quality was observed.

The following figure shows the relationship between SF and SIRT in terms of probability density function (PDF). 


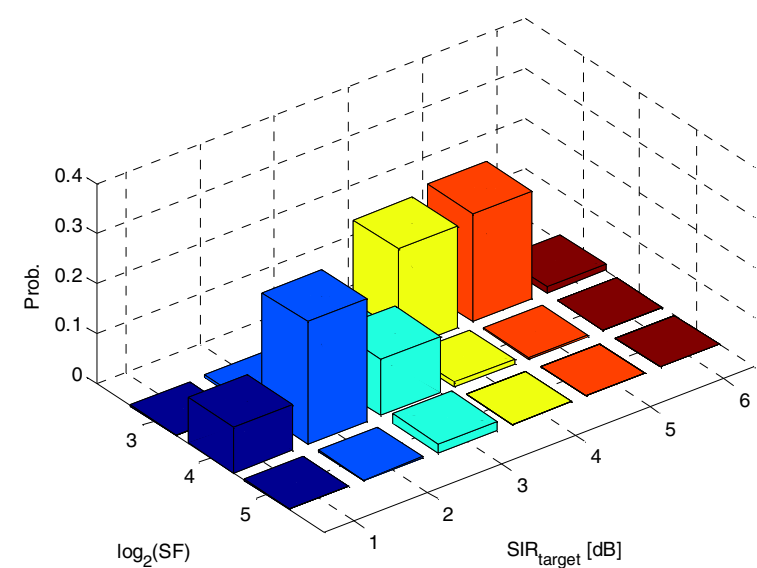

Figure 4 Relationship between SF and SIRT

Figure 4 indicates that a low SIRT around $2 \mathrm{~dB}$ is sufficient for a code with high SF $\left(2^{4}=16\right)$, while a higher SIRT at around $4-5 \mathrm{~dB}$ is required when $\mathrm{SF}=8$ is in use.

\subsection{Mobile Live TV: Cell Edge}

In this section, the WCDMA performance of a case when a terminal using a real-time video streaming application is moving out of coverage area is studied.
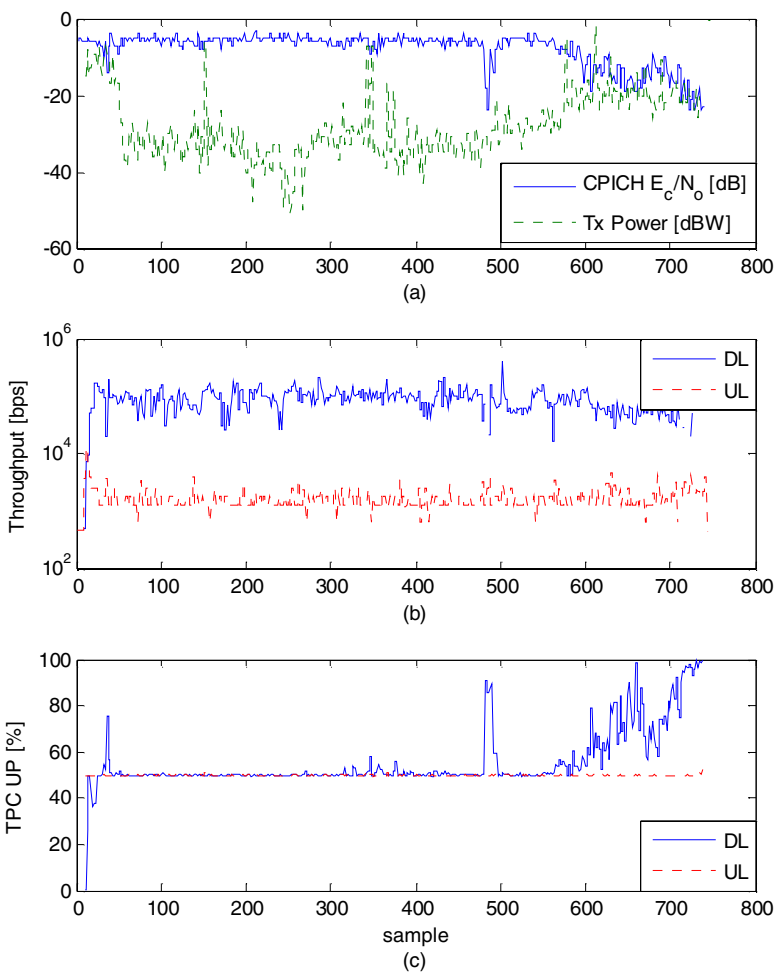

Figure 5 WCDMA performance at a cell edge

Figure 5 (a) $\mathrm{CPICH} \mathrm{Ec/No} \mathrm{and} \mathrm{transmission} \mathrm{power}$ when a UE is moving out of coverage. As the terminal was forced to connect to a base station, which might not be the best one, the transmission power required to maintain this connection might be higher than that in Section 3.1. From this figure, the UE seems to move out of coverage from the sample $600^{\text {th }}$ onward. This expectation is consistent with Figure 5 (c) in which a large number of TCP power up commands are issued.

In Figure 5 (b), it is interesting to see that: although the UE was out of coverage, video data could be streamed to the end user. This is due to the fact that real-time services such as real-time video are, in most cases, based on a realtime protocol. Such real-time protocol is often an "unacknowledged" protocol. This means the data are still sent even if the receiver could not receive them or if received in error. This situation is WCDMA is illustrated in Figure 6.
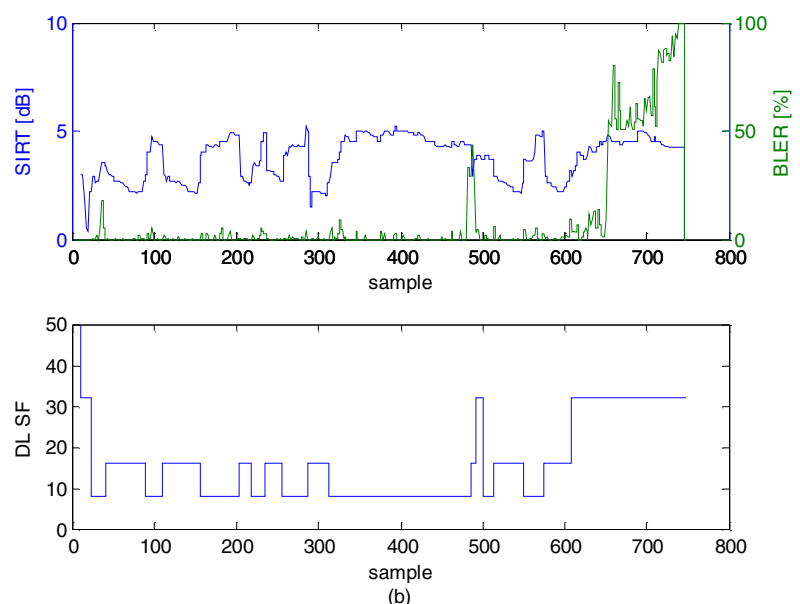

Figure 6 WCDMA adaptive parameters at cell edge

In Figure 6 , the results in samples $0^{\text {th }}$ to $600^{\text {th }}$, show a similar trend as that shown in Figure 3. There are two major differences between these two figures. The first difference occurs around the $500^{\text {th }}$ sample. In this period, the $\mathrm{CPICH}$ Ec/No suddenly drops as shown in Figure 5 (a). The mechanism that RNC uses to handle this drop was interesting, ie. the RNC decided to keep SIRT at approximately the same level but increases SF by two levels, from 8 to 32. This resulted in lower transmission power required at $\mathrm{UE}$, but poorer quality of real-time video that the user was experiencing. During this period, many pauses and buffering were observed by the user.

Another major different that must be noted from Figure 3 and Figure 5 is when the UE is moving out of coverage area (Figure 5 and 6 from the $600^{\text {th }}$ samples onward). Figure 5 (b) shows that the DL throughput from the $600^{\text {th }}$ sample onwards is approximately a half $(50 \mathrm{kbps})$ of typical required throughput for video streaming service (around $100 \mathrm{kbps}$ ). As the terminal moved further away from the base station, this $50 \mathrm{kbps}$ data rate could be delivered to the end user. However, a large BLER of more than $50 \%$ was received as shown in Figure 6 (b). As a result, proper communication is not possible. 
The relationships between SF and SIRT for the $51^{\text {st }}$ to $600^{\text {th }}$ and $601^{\text {st }}$ to $748^{\text {th }}$ samples are shown in Figure 7 and 8 , respectively.

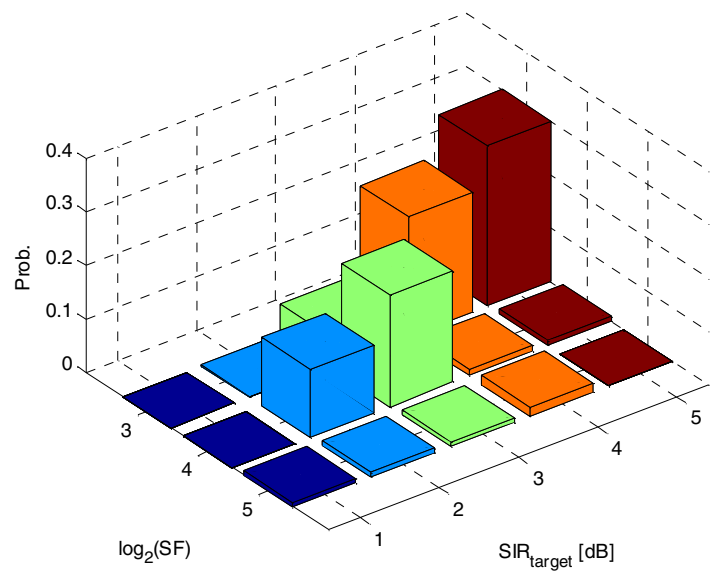

Figure $7 \mathrm{SF}$ and SIRT for the $51^{\text {st }}$ to $600^{\text {th }}$ samples

Figure 7 shows the same trend as Figure 4 . The only difference is a higher level of SIRT on average is required in Figure 7. This is due to the fact that the situation of Figure 7 was when the terminal was forced to connect to a base station which might not be a base station that provided the best signal. RNC might detect that the radio condition between the serving Node B and the UE is poor, and hence it increased the SIRT level.

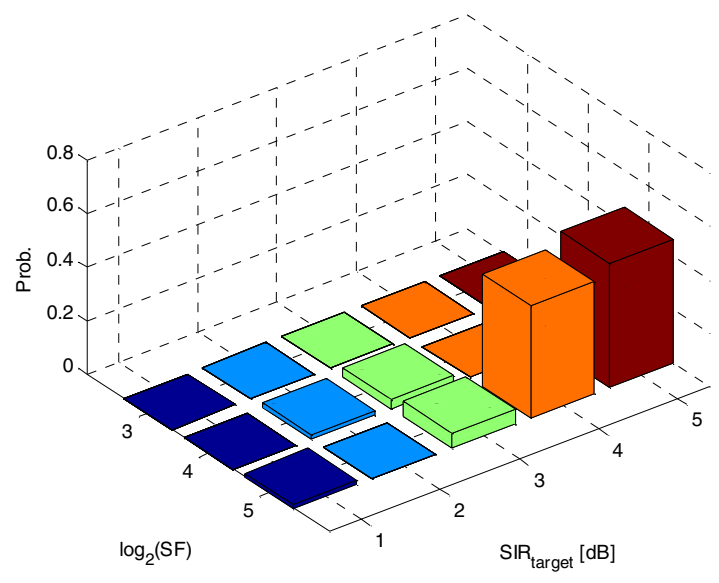

Figure $8 \mathrm{SF}$ and SIRT for the $601^{\text {st }}$ to $748^{\text {th }}$ samples

Figure 8 together with Figure 5 (a) and (b) clearly show that when a terminal is moving out of coverage, the transmission power and SIRT required to maintain the connection are high, but the throughput of the service decreases.

\section{Conclusion}

This paper presents experimental results when a user is using a real-time video streaming application in a commercial UMTS network in Australia. Two scenarios are considered: a typical scenario when soft handover between base stations is allowed, and an extreme scenario when a terminal is moving out of coverage. The experiments illustrate the practical implementation of protocols used for real-time TV applications. The results also shown that, when the channel quality is poor, video data are still streamed to the user even when BLER is significantly increasing. In such situation, RNC decides to use a code with high SF which requires low SIRT to avoid increasing UE transmission power. These match well with the theory.

\section{Future Work}

This study is limited to the performance of streaming services in two scenarios only. It would be interesting to study the performance of such services in other situations such as when a user is walking and/or on a vehicle. Another potential research is to study similar performance and implementation metrics of other types of services such as downloading.

\section{Acknowledgement}

The authors would like to grateful thank to the CRIN to support this work.

\section{Reference}

[1] F. Falcone, I.D. Escauriaza, A.V. Fernandez, F. B. Mau, "Performance Analysis by Measurement Results in Operating 3G Network", in Proceeding of The 3rd International Symposium on Wireless Communication Systems, (ISWCS '06), pp. 671 - 673, 6-8 Sept. 2006.

[2] M. Da Silva, Y. Farmine, "W-CDMA uplink soft handover gain measurements", in Proceeding of the Vehicular Technology Conference, VTC 2005, Vol. 1, pp. 416- 418, 30 May-1 June 2005.

[3] N. Kara, O. Issa, A. Byette "Real 3G WCDMA networks performance analysis", in Proceeding of the 30th IEEE Conference on Local Computer Networks, pp. 1-7, 15-17 Nov. 2005

[4] K. J. Grandell, O. Salonaho, "Macro cell measurements with the Nokia WCDMA experimental system", in Proceeding of the Eleventh International Conference on Antennas and Propagation, Manchester, UK, Vol. 2, pp. 516-520, 17-21 April 2001.

[5] M. Etoh and T. Yoshimura, "Wireless video applications in $3 \mathrm{G}$ and beyond", IEEE Wireless Communications, Vol. 12, No. 4, August, 2005, Advances in Wireless Video, p 66-72

[6] Laibo, J., A. Wacker, and T. Novosad; Radio Network Planning and Optimization for UMTS. Wiley, New York, 2006. 

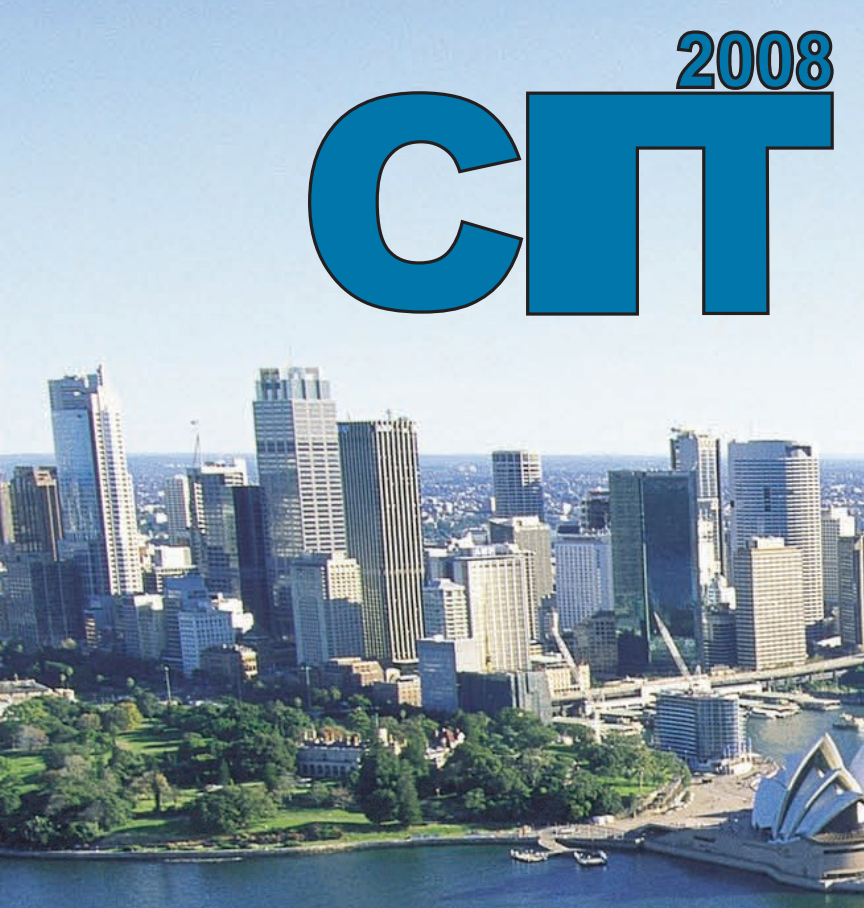

\section{IEEE 8th International Conference on Computer and Information Technology \\ 8-11 July 2008 - Sydney, Australia}

Hub Page

Table of Contents

Author Index

Search

Support

Install Software

\section{Proceedings of}




\title{
C. \\ Hub Page
}

\section{IEEE $8^{\text {th }}$ International Conference on Computer and Information Technology}

\author{
8-11 July 2008 - Sydney, Australia
}

\begin{tabular}{|l|}
\hline Welcome Page \\
\hline Welcome from Chairs \\
\hline Organizing Committee \\
\hline Program Committee \\
\hline Additional Reviewers \\
\hline
\end{tabular}

\begin{tabular}{|l|}
\hline Session List \\
\hline Table of Contents \\
\hline Author Index: Brief or Detailed \\
\hline Manuscripts \\
\hline
\end{tabular}

Proceedings of the 2008 IEEE 8th International Conference on Computer and Information Technology. IEEE Catalog Number CFP08355-CDR, ISBN 978-1-4244-2358-3, Library of Congress 2008902859. Copyright (c) 2008 IEEE. Personal use of this material is permitted. However, permission to reprint/republish this material for advertising or promotional purposes or for creating new collective works for resale or redistribution to servers or lists, or to reuse any copyrighted component of this work in other works must be obtained from the IEEE. For technical support please contact Causal Productions (info@causalproductions.com).

\section{Legend: View Manuscript}


Networking and Mobile Computing
607 TCP-Friendly Streaming Video System with Error-Resilient Coding Over Wireless Vertical Handover Environment
(Jae-Young Pyun, Ho-Jin Choi)

613 Enhancement of PAPR Reduction Using Dynamic PTS Algorithm in Multiple Antennas System (Guo-fang Dong, Fei Gao, Jing Fan, Zi-bin Zhao)

618 Challenges and Recent Advances in QoS Provisioning in Wireless Mesh Networks (Shivanajay Marwaha, Jadwiga Indulska, Marius Portmann)

624 Performance Analysis of an Adaptive Backoff Scheme for Ad Hoc Networks (Jing Fan, Fei Gao, Wan-Sheng Wang, Guo-fang Dong)

630 Performance Study of Video Streaming in Commercial UMTS Network (Rachod Patachaianand, Kumbesan Sandrasegaran)

634 An Approach to Select the Best Spanning Tree in Metro Ethernet Networks (Ghasem Mirjalily, Mohammad Hadi Karimi, Fazlollah Adibnia, Shahram Rajai)

640 Cooperative Channel Operation MAC Protocol for WLAN Based Indoor Cognitive Radio Network Systems

(Nan Hao, Jae-Kark Choi, Sang-Jo Yoo)

646 Fast Group Scanning Scheme with Dynamic Neighbor Base Station List in IEEE 802.16e Networks

(Jae-Kark Choi, Nan Hao, Sang-Jo Yoo)

652 Fuzzy Logic Based Modelling and Analysis of Network Traffic

(A. Rahman, P. Kennedy, A. Simmonds, J. Edwards)

658 Towards Fair P2P Auctions Over MANETs

(Ines Doghri, Hella Kaffel-Ben Ayed) 


\section{Proceedings}

\section{IEEE 8th International Conference on Computer and Information Technology}

\section{CIT 2008}

8-11 July $2008 \cdot$ Sydney, Australia

\section{Editors}

Qiang Wu, Xiangjian He, Quang Vinh Nguyen, Wenjing Jia and Maolin Huang

Organized by

CIT Organizing Committee

In cooperation with

IEEE Computer Society

IEEE Technical Committee on Scalable Computing

University of Technology, Sydney

Research Institute for Information and Communication Technology,

Korea University, Korea

BK 21 Information Technology Division, Korea University, Korea

ARC Research Network in Enterprise Information Infrastructure (EII), Australia

Federation of Chinese Scholars in Australia (FOCSA), Australia

Australian Chinese ICT Professional Society, Australia 